\title{
Transparent Motion Perception as Detection of Unbalanced Motion Signals. II. Physiology
}

\author{
Ning Qiana and Richard A. Andersen \\ Department of Brain and Cognitive Sciences, Massachusetts Institute of Technology, Cambridge, Massachusetts 02139
}

\begin{abstract}
We investigated how the primate visual system solves the difficult problem of representing multiple motion vectors in the same part of the visual space-the problem of motion transparency. In the preceding companion article we reported that displays with locally well-balanced motion signals in opposite directions are perceptually nontransparent (i.e., one does not see two coherent moving surfaces) and that transparent displays always contain locally unbalanced motion signals. This is exemplified by our paired and unpaired dot patterns. Although both types of stimuli contain two sets of dots moving in opposite directions, the former is locally well balanced and appears like flicker while the latter gives a perception of two transparent surfaces. In this article we report our physiological recordings from areas $V_{1}$ and MT of behaving monkeys, comparing single-cell responses to the paired and the unpaired dot patterns. Although a small proportion of directionally selective V1 cells responded differently to the two types of patterns, the average $V 1$ responses could not rellably distinguish between the paired and the unpaired stimuli. A large fraction of MT cells, on the other hand, responded significantly better to the unpaired dot patterns than to the paired ones. Furthermore, the average response of all MT cells to the unpaired dot patterns was significantly higher than that to the paired dot patterns.
\end{abstract}

These results demonstrate a neural correlate of the perceptual transparency at the level of MT. On the other hand, $V 1$ cells do not generally discriminate between the transparent and nontransparent stimuli, indicating that $V 1$ activity is not well correlated with the perception of motion transparency. Our results are consistent with a two-stage model for motion processing: the first stage measures local motion and the second stage introduces suppression if different directions of motion are present at a local region of the visual field. The first stage is located primarily in $\mathrm{V} 1$ and the second stage primarily in MT. Finally, we found a strong and negative correlation between the degree of the opponent-direction suppression of MT cells and their responses to flicker noise

\footnotetext{
Received Aug. 18, 1993; revised Apr. 18, 1994; accepted Apr. 27, 1994.

We thank Dr. Stefan Treue for many helpful discussions and two anonymous reviewers for their insightful comments. The research is supported by Office of Naval Research Contract N00014-89-J1236 and NIH Grant EY07492, both to R.A.A. N.Q. was supported by a McDonnell-Pew postdoctoral fellowship during the early phase of this work.

Correspondence should be addressed to Dr. Richard A. Andersen, Division of

Biology, 217-76, California Institute of Technology, Pasadena, CA 91125.

Present address: Center for Neurobiology and Behavior, Columbia University, New York, NY 10032.

Copyright (C) 1994 Society for Neurcscience $0270-6474 / 94 / 147367-14 \$ 05.00 / 0$
}

stimuli. This result suggests that one of the fundamental roles of the opponent-direction suppression in MT is noise reduction.

[Key words: motion transparency, behaving monkey physiology, area MT, area V1, opponent-direction suppression, directional selectivity]

Our visual system can represent more than one motion in the same part of visual space. For example, when looking at a display composed of two independent sets of random dots moving in opposite directions in the same part of a video monitor, we usually see two transparent surfaces moving continuously across each other. This phenomenon is an example of transparent motion perception. Examples of motion transparency that occur frequently in the real world are partial occlusions or semitransparent surfaces in a scene with moving objects.

In the preceding companion article, we studied a series of visual patterns, which all had two components moving in opposite directions, in order to determine the conditions under which transparent motion perception occurs (Qian et al., 1994a). We found that displays with locally well-balanced motion signals in opposite directions are perceptually nontransparent and that transparent displays always contain locally unbalanced motion signals in different directions. The best examples are the paired and the unpaired random dot patterns we generated. A paired dot pattern is composed of many randomly located pairs of dots. The two dots in each pair move across each other in opposite directions over a short distance and then jump to a new random location to repeat the process. In the corresponding unpaired dot pattern, the dots moving in opposite directions are simply unpaired and positioned independently. The two types of displays are identical in all aspects except that there is pairing in one type but not in the other. While the unpaired dot patterns give the percept of two transparent surfaces moving across each other, the paired dot patterns do not and look rather like flicker. We explained these results by proposing an opponent or suppression stage in the motion pathway at which motion signals from different directions of motion locally inhibit each other (Qian et al., 1994a; see also following companion article, Qian et al., 1994b). For the paired dot patterns there are precisely two opposite motion vectors at a given location so they strongly cancel each other at the suppression stage. On the other hand, the unpaired dot patterns contain locally unbalanced motion signals in oppositc directions duc to the local dot density fluctuations and cancellation at the suppression stage would consequently be much weaker. For example, in a small area there might be two dots moving to the left and only one to the right. Different small areas of the display can contain net motion 
signals in one of the two opposing directions. These spatially mixed net motion signals in both directions could later be integrated separately to form two overlapping transparent surfaces.

In this article, we report our physiological investigation of the proposed suppression stage. A previous study from our laboratory (Snowden et al., 1991) indicates that MT cells show strong inhibition between different directions of motion. MT is therefore a good candidate for the suppression stage we proposed. In the present study, we tested this hypothesis directly through single-unit recordings from area MT of behaving monkeys, using the paired and the unpaired dot patterns. We also report results from V1 recordings using the same set of stimuli for comparison.

The second issue we addressed in this study is why there should be a suppression stage in the motion pathway in the first place, since it is certainly not just for making the locally wellbalanced patterns, such as the paired dot patterns, appear nontransparent. We propose that an important functional role of the directional suppression in MT is to reduce response to motion noise. An example of motion noise is flicker stimuli, which radiates motion energy in all directions. We tested this hypothesis by recording V1 and MT cells' responses to noise patterns made of flickering random dots.

Part of the results has been presented previously in abstract forms (Qian et al., 1991, 1992).

\section{Materials and Methods}

Preparation of animals. Most data reported in this article were obtained from two male rhesus monkeys (Macaca mulatta). Two additional male rhesus monkeys were also used in a few recording sessions. Details of the training and experimental procedures have been published previously (Snowden et al., 1991). Briefly, the animals were trained to fixate for several seconds in a dimming-detection reaction-time task. The animals' heads were fixed throughout the experiments and their eye positions were monitored during the fixation period by the magnetic search coil technique (Robinson, 1963). The standard deviations of the horizontal and vertical eye positions on the successful trials for the first monkey were $3.5 \mathrm{~min}$ and $4.9 \mathrm{~min}$, respectively. Those for the second monkey were $3.8 \mathrm{~min}$ and $4.0 \mathrm{~min}$, respectively. The recording chamber was placed over area V1 such that area MT was accessed with deep penetrations through $\mathrm{V} 1$.

Stimuli. We developed an integrated software package for our physiological experiments. All displays (motion stimuli, a fixation point, and a bar for mapping receptive fields) were generated on a AST 386 PC with a Number Nine SGT graphics board installed. The vertical refresh rate of the board was $60 \mathrm{~Hz}$ noninterlaced. The output of the board was sent to two video monitors simultaneously, one (Monitronix) in front of the monkey in a closed and dimly lit experiment room, and the other outside in the control area for the experimenters. The Monitronix monitor was calibrated with an EG\&G Gammer Scientific photometer (model 450-1) with capacitors added across the output resistors in order to integrate over the monitor's refresh period to obtain time-averaged readings. The fixation point, the bar for receptive field mapping, and the visual stimuli were all under the control of a Microsoft mouse and could be moved to any location on the monitor screen. Using various mouse and keyboard combinations, we could also change the sizes of the fixation point and of the stimuli, and modify the width, height, and orientation of the bar. In most of our experiments, the monitor was 57 $\mathrm{cm}$ away from the monkeys' eyes. At this distance, each pixel extends a visual angle of $0.056^{\circ}$.

All stimuli used for MT recordings covered a round area of $3^{\circ}$ in radius, and each lasted for $1 \mathrm{sec}$. The dots in the stimuli had a lifetime of 7 frames $(117 \mathrm{msec})$ before being replotted. In order to measure the directional tuning of cells, we generated a set of eight stimuli; each consistcd of 100 randomly located dots moving in one of the eight directions. These eight directions define four axes of motion: one vertical, one horizontal, and two at $45^{\circ}$ oblique angles. We then generated four paired and four unpaired dot patterns along the same axes of motion. There were 200 dots in each of these patterns, with 100 of them moving in one direction, and the othcr 100 moving in the oppositc direction. A paired dot pattern contained 100 pairs of dots. The two dots in each pair started at a separation of 7 pixels $\left(0.4^{\circ}\right)$. They moved toward and across each other, and were replotted when the separation was again 7 pixels. The replotting of the dot pairs was asynchronized with respect to each other. The unpaired dot patterns were identical to the paired ones except that the two dots in each pair were positioned independently and randomly. All dots moved at a speed of 1 pixel/ frame or $3.36 \% \mathrm{sec}$. We also generated two flicker noise patterns, one derived from the paired dot patterns and the other from the unpaired dot patterns. They were identical to the moving patterns in all aspects except none of the dots moved from frame to frame. Each dot simply stayed in a fixed random location during its lifetime ( 7 frames or 117 $\mathrm{msec}$ ) and then was replotted at a new random location.

The above sct of stimuli (the standard sct) was also used in most of our $\mathrm{V} 1$ recordings. However, since $\mathrm{V} 1$ cells have much smaller receptive field sizes than MT cells we also generated a set of control patterns with radius equal to $1.5^{\circ}$ and the rest of the parameters identical to those of the standard set. The dot density of the control patterns is thus four times as high as in the standard patterns. The detailed discussion of the issues related to spatial scale difference between V1 and MT will be presented in the text.

During data collection, two stimuli were presented one after another in a single trial, with a blank interval between them. We arranged the above mentioned 18 stimuli (eight stimuli with single sets of moving dots, four paired dot patterns, four unpaired patterns, one paired flicker, and one unpaired flicker) into nine classes, with two stimuli in each class. For the stimuli with single sets of moving dots, the two displays with dots moving in the opposite directions form a class. For the paired and the unpaired dot patterns, the two (one from each type) with the same axis of motion were put in one class. The paired and the unpaired flicker noise patterns constitute the final class. These nine classes formed a single block during recording and were presented in a pseudorandom order. While this arrangement of two stimuli into one trial significantly increased the speed of data collection, it also introduced a potential bias: the paired patterns always came before the unpaired ones in each trial. As a control, we also arranged the 18 stimuli into a different block of nine classes. The second block was identical to the first one except the order of the paired and the unpaired patterns in each class was switched. We used each one of the two blocks on approximately half of the cells recorded. Some other control stimuli were also generated and they will be mentioned in the text.

Recording procedure. The experiment was controlled by a PDP-11 computer, which monitored the monkey's behavior (eye position, pulling and releasing of the key), sent signals to the PC for displaying the fixation point and the stimuli, collected data, and gave juice reward to the monkey. Early MT recordings were made with glass-coated platinum-iridium electrodes. We later switched to tungsten electrodes with guide tubes. All V1 recordings were performed with the glass-coated platinum-iridium electrodes. After a cell was isolated, we first attempted to map its receptive field with mouse-controlled bars of different sizes, orientations, and speeds while the monkey performed the fixation task. For a small proportion of cells that could not be well driven by the bar stimuli, a mouse-controlled small random dot pattern with adjustable size was used instead. After the receptive field border was approximately determined, the stimuli were positioned in its center. Then, a series of blocks of trials were presented until all experiments were completed or until the cell was lost. For the V1 cells we recorded, the receptive field diameters were normally below $0.5^{\circ}$, considerably smaller than our stimuli. Most MT cells we isolated, on the other hand, had receptive field sizes ranging from $6^{\circ}$ to $10^{\circ}$, and the stimuli were inside the classical receptive fields (Allman et al., 1985).

Data collection and analysis. The data were collected from the periods when the monkeys were fixating and various displays were shown in the cells' receptive fields. The time intervals between successive spikes were recorded. In each trial, the collection started after the monkeys established fixation; $500 \mathrm{msec}$ later, a $1 \mathrm{sec}$ stimulus appeared, followed by a blank interval of $1.1 \mathrm{sec}$, and then another $1 \mathrm{sec}$ stimulus. During data collection, a spike raster was displayed after the completion of each trial and the corresponding histogram was updated. The data were saved in a filc after cach block of trials for off-line analysis on a Macintosh computer. The first $200 \mathrm{msec}$ of the responses to stimuli was excluded from quantitative analysis to avoid the bias of the transient effects. In most blocks there is also a class of trials in which no stimulus was shown. The activities of the cells during these "fixation-alone" trials 


\section{MT Cell}
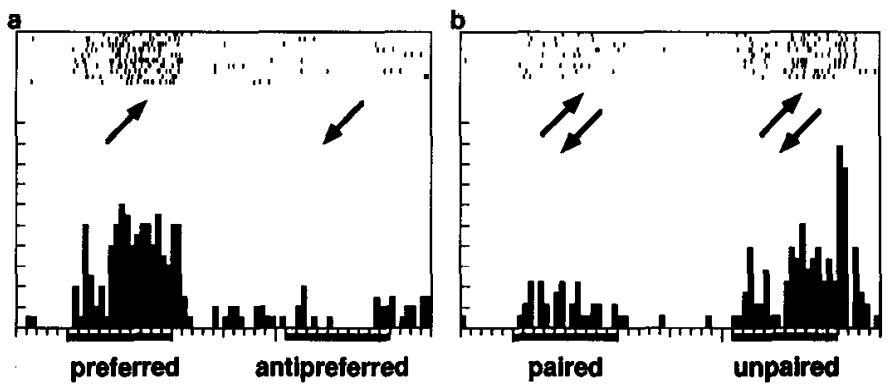

Figure I. An MT cell with significantly stronger response to the unpaired dot pattern than to the paired dot pattern. $a$, Responses to single sets of random dots moving in its preferred and the antipreferred directions. $b$, Responses to the paired and the unpaired dot patterns with one set of dots moving in the preferred direction and the other the antipreferred direction. The raster at the top of each diagram represents the spike records from several repeated trials. Each small dot in the raster represents the occurrence of a spike. The response histograms compiled from the rasters are shown at the bottom of the diagrams. The arrows below the rasters indicate the directions of motion. Each small division in the horizontal axis represent $10 \mathrm{msec}$. The $1 \mathrm{sec}$ periods during which the stimuli were presented are marked by the thick black lines under the histograms. One small vertical division represents 4.0 spikes/sec.

were used to calculate their spontaneous rates of firing. For a small number of cells from which we did not collect data with fixation point alone, we used the activities in the last $0.5 \mathrm{sec}$ of the blank interval between the two stimuli in all trials to calculate the background rate. The cells were first screened based on their reliability to fire above the spontaneous rate in at least one direction of motion. A two-tailed $t$ test with a significance level of $5 \%$ was used in the screening process. For cells that passed this test, the direction with the highest firing rates was considered as their preferred direction of motion, and the opposite direction the antipreferred direction. They were then tested for their directionality by comparing their firing rates along the preferred and the antipreferred directions. Again, a two-tailed $t$ test with a significance level of $5 \%$ was used. Only the directionally selective cells were included in our analysis.

\section{Results}

\section{$M T$ responses to paired and unpaired dot patterns}

We first report MT responses to the paired and the unpaired dot patterns. Previous physiological studies from our laboratory indicate that MT cells show strong suppression when different directions of motion are present simultaneously (Snowden et al., 1991). On the other hand, V1 cells generally are not as suppressed under these conditions. MT is therefore likely to be the suppression stage that could distinguish the paired and the unpaired dot patterns by measuring the amount of locally unbalanced motion signals in these displays (Qian et al., 1994a). If this is true, then two predictions can be made. First, MT cells' responses to both the paired and the unpaired dot patterns (with one set of dots moving in their preferred directions and the other in their antipreferred directions) should be reduced in comparison with the responses to single sets of dots moving in their preferred directions alone. This is simply because the presence of the sets of dots in the antipreferred directions should always generate a certain amount of suppression. Second, and more importantly, the amount of suppression should be stronger (i.e., the response smaller) for the paired dot patterns than for the unpaired ones, because the opposing motion signals in the
MT Cell
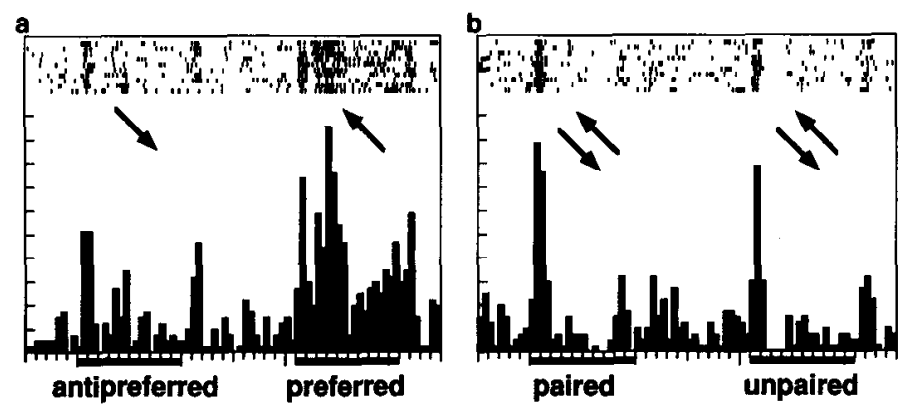

Figure 2. An MT cell with equal responses to the paired and the unpaired dot pattern. See Figure 1 for details. One small vertical division represents 8.2 spikes $/ \mathrm{sec}$.

paired dot patterns are much better balanced and therefore cancel each other more completely.

We indeed found many MT cells that behaved as predicted. An example is shown in Figure 1. Figure 1a shows the cell's responses to single sets of dots moving in its preferred and the antipreferred directions, respectively. It is evident that the cell is highly directionally selective, which is typical for MT cells. Figure $1 b$ shows the cell's responses to the paired and the unpaired dot patterns. Both responses are weaker than the preferred direction response and the response to the paired dot pattern is significantly weaker than that to the corresponding unpaired dot patterns. We also found many cells that did not show significant differences in their responses to the paired and the unpaired dot patterns. An example is shown in Figure 2. Finally, there is a very small fraction of cells that showed the opposite behavior from what we predicted; that is, their responses to the paired dot patterns are larger than to the unpaired dot patterns. Figure 3 gives an example. We will later discuss the differences among these three categories of cells in more detail.

To see if the overall behavior of MT cells is consistent with our predictions, we recorded a total of $91 \mathrm{MT}$ cells and the population results are summarized in Figure 4. To characterize the cells quantitativcly, we compute two suppression indices for each cell, one for the paired dot pattern and the other for the unpaired dot pattern along the preferred-antipreferred axis of motion. They are defined as

$$
\begin{gathered}
S I_{\mathrm{p}}=1-\frac{\text { paired response }}{\text { preferred response }} \\
S I_{\mathrm{up}}=1-\frac{\text { unpaired response }}{\text { preferred response }} .
\end{gathered}
$$

The background firing rate was subtracted from all responses before calculation. These indices represent the percent reductions of a cell's responses to the paired and the unpaired dot patterns, respectively, in comparison with its preferred direction response. They therefore measure the degrees of suppression between preferred and antipreferred directions of motion. An index near zero indicates no suppression, a large value indicates strong suppression, and a negative value means that enhancement instead of suppression has occurred.

Figure 4 plots, for each cell, the unpaired suppression index $\mathrm{SI}_{\mathrm{up}}$ against the paired suppression index $\mathrm{SI}_{\mathrm{p}}$. First note that the 


\section{MT Cell}
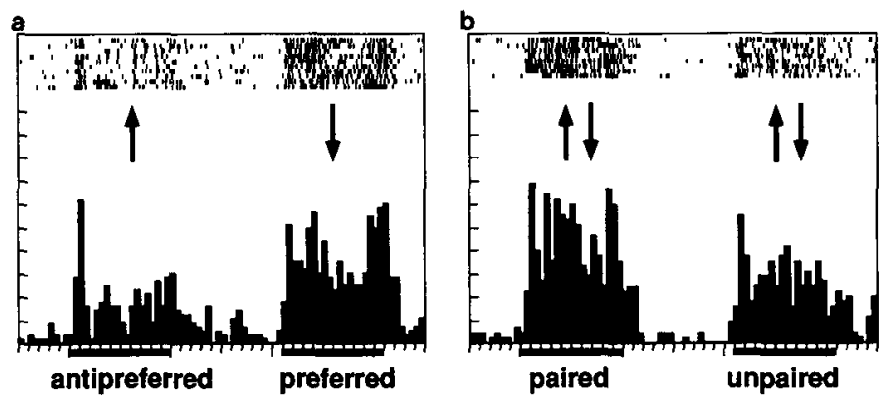

Figure 3. An MT cell with significantly stronger response to the paired dot pattern than to the unpaired dot pattern. See Figure 1 for details. One small vertical division represents 11.3 spikes $/ \mathrm{sec}$.

suppression indices for almost all MT cells, whether measured with the paired or the unpaired dot patterns, are positive. This can be seen by projecting the dots in Figure 4 along either coordinate axis. The result agrees with our previous finding that MT cells show significant suppression among different directions (Snowden et al., 1991).

We next examine how cells responded differently to the transparent and the nontransparent patterns. Cells with similar responses (thus equal degrees of suppression) to the paired and the unpaired dot patterns lie near the diagonal line. These cells cannot distinguish the transparent patterns from the nontransparent ones. Those falling well below the diagonal line show stronger suppression (or less responses) for the paired dot patterns than for the unpaired ones. Finally, cells well above the diagonal line have the opposite behavior: they show stronger suppression for the unpaired dot patterns than for the paired ones. There are significantly more cells below the diagonal line than above in Figure 4 (Wilcoxon signed-rank test, $p=0.0001$ ), consistent with our prediction.

We next consider each cell individually. We performed a twotailed $t$ test for each cell to see if its mean response to the unpaired dot pattern over repeated trials is significantly different from that to the paired dot pattern. The cells that did show significant difference between the two patterns are plotted as solid dots while those did not as open dots in Figure 4. We found that $40 \%$ of cells responded significantly stronger to the unpaired dot patterns than to the paired dot patterns, while only $10 \%$ displayed the opposite behavior. The remaining $50 \%$ did not show significant differences between the two types of displays. We will give an explanation for the behavior of the cells in the last category later. It is also worth pointing out that we classified cells into the above three categories only for the convenience of presentation. The population of MT cells forms a continuum instead of discrete classes.

One possible cause of the difference between a cell's response to the paired and the unpaired dot patterns is that there might be some "hot spots" in the cell's receptive field, so that one pattern might happen to contain some dots moving across these spots while the other misses them. This is, however, unlikely because there are 100 dots moving in each of the two directions, and MT cells are generally saturated when there are just 5 dots in their receptive fields (Snowden et al., 1991). Also, during the $1 \mathrm{sec}$ display period of a stimulus, each dot was replotted more than eight times to a new random location within the receptive

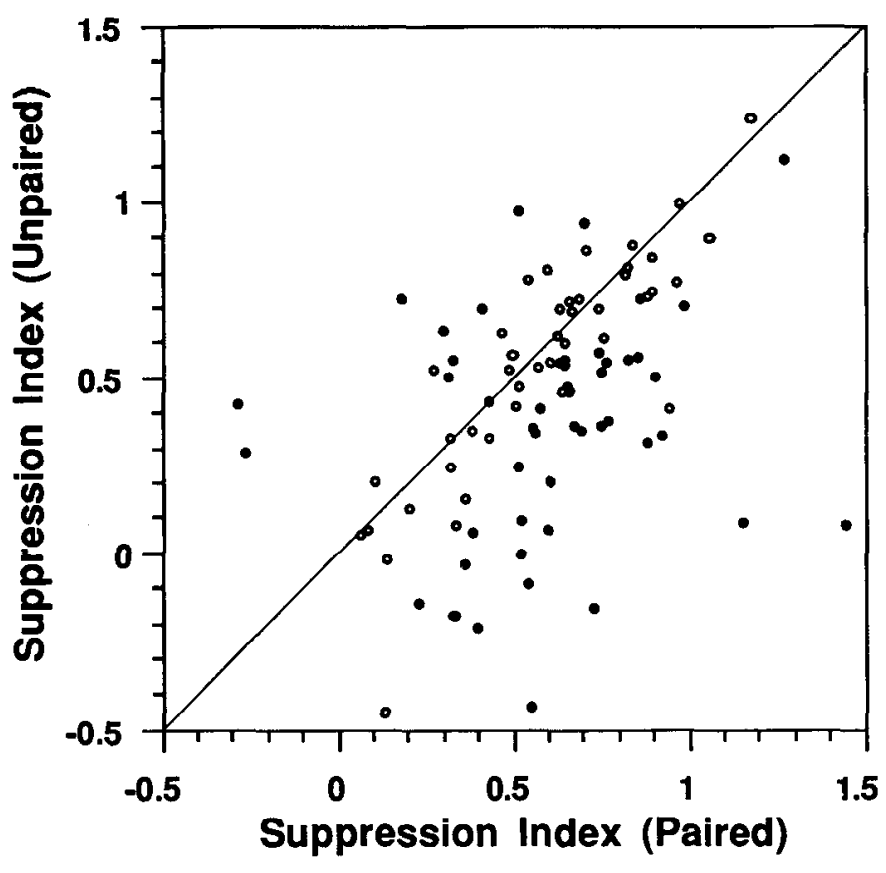

Figure 4. The suppression index for the paired dot pattern of each MT cell is plotted against its suppression index for the unpaired dot pattern. The cells with significantly different responses to the two types of patterns based on a two-tailed $t$ test are shown as solid circles. The rest are shown as open circles.

field (see Materials and Methods). This makes the dot distribution much more uniform. Furthermore, this possibility would generate about equal numbers of cells with the predicted and the opposite behavior and therefore could not explain the bias toward the weaker suppression (stronger responses) for the unpaired dot patterns in Figure 4. We nevertheless did a control to rule out this possibility. Along each axis of motion, we generated independently four more paired and four more unpaired dot patterns using the same sets of parameters. All of the five paired (or the unpaired) dot patterns were statistically and perceptually identical, but the exact spatial and temporal locations of dots in these patterns were different because of the random nature of dot generation. We tested cells' responses on all of these paired and the unpaired dot patterns to see if there is consistency in their responses. An example of a cell tested this way is shown in Figure 5. In Figure 5, $a$ shows the cell's preferred and antipreferred responses, and $b-f$ show that its responses to the five sets of independently generated paired and the unpaired dot patterns. This cell responded better to the unpaired dot patterns than the paired ones in all cases. For eight cells tested this way, seven showed consistent responses to all five sets of patterns. Only one cell had a little variation: its responses to the paired and the unpaired dot patterns were different for one set of patterns, but identical for the remaining four sets.

Another concern is that during the lifetime ( 7 frames) of a dot in a paired dot pattern, there is one frame in which the two dots in a pair overlap. The overlapping in the unpaired dot patterns, on the other hand, occurs only by chance and thus much less frequently. We did not double the luminance when two dots overlapped. We performed a control with a new type of paired dot patterns, in which an offset of 1 pixel perpendicular 


\section{MT Cell}
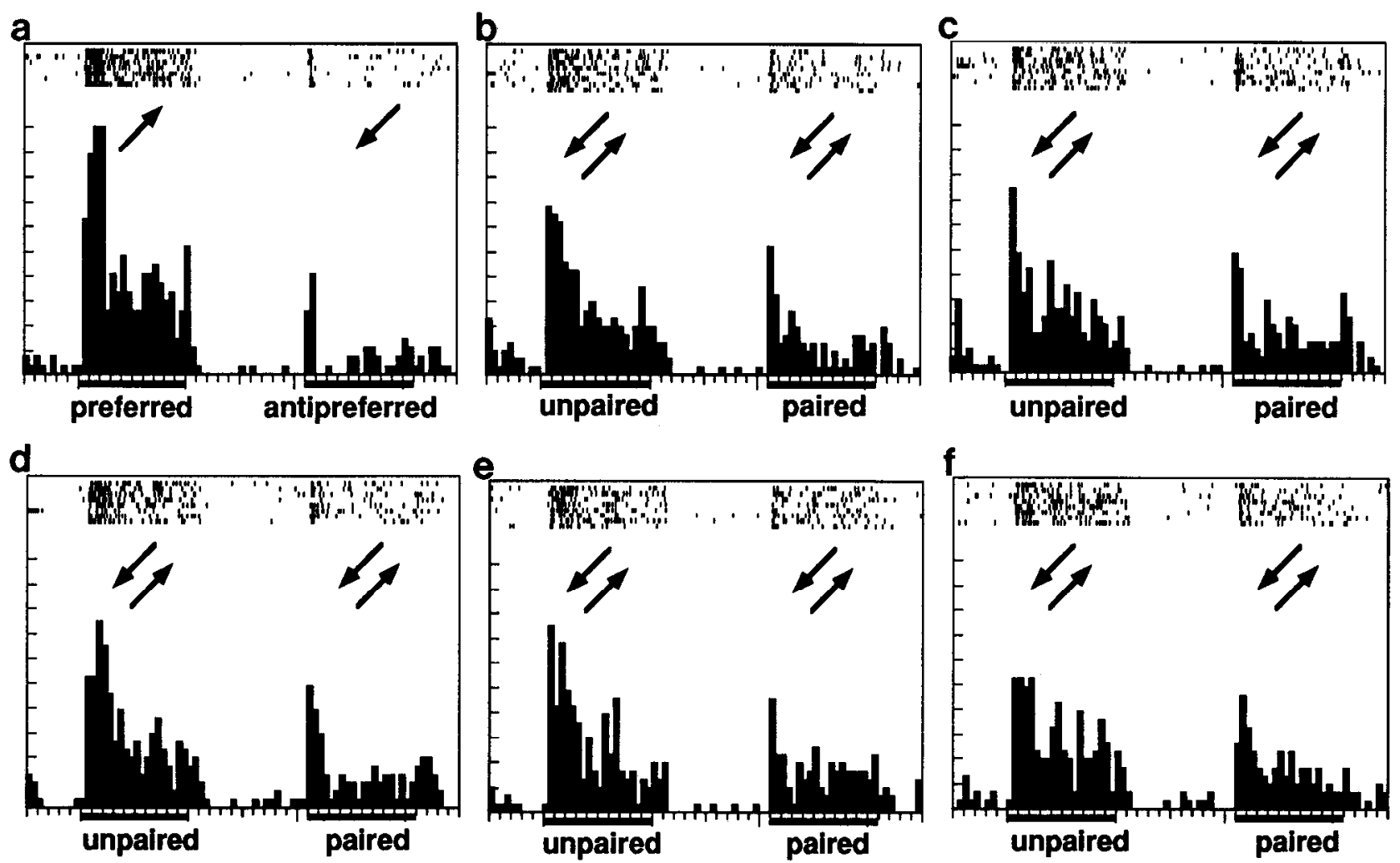

Figure 5. An MT cell tested for consistency on five sets of independently generated paired and unpaired dot patterns. $a$, Responses to single sets of random dots moving in its preferred and the antipreferred directions. $b-f$, Responses to five sets of independently generated paired and unpaired dot patterns with one set of dots moving in the preferred direction and the other the antipreferred direction. One small vertical division represents 7.7 spikes $/ \mathrm{sec}$.

to the axis of motion was introduced between the two dots in each pair. Our previous psychophysical observations indicate that the paired dot patterns with 1 pixel vertical offset are perceptually similar to the standard paired dot patterns, and that both are much less transparent than the unpaired dot patterns (see Qian et al., 1993a, Fig. 3). A cell tested this way is shown in Figure 6. It responded better to the unpaired dot pattern than to the paired one with offset. Among 21 MT cells tested, 12 responded significantly stronger to the unpaired dot patterns while only one cell responded better to the paired dot patterns. The rest did not show a significant difference. These results are consistent with those reported above using the paired dot patterns without offset.

\section{MT responses to noise patterns}

We have shown above that MT cells exhibited suppression between opposite directions of motion and $40 \%$ of them showed stronger suppression for the paired dot patterns than for the unpaired ones. These results are consistent with our prediction that there is an opponent or suppression stage in the motion pathway at which motion signals from different directions of motion locally inhibit each other, and that cells in this stage could distinguish the perceptually transparent patterns from the nontransparent ones by measuring the locally unbalanced motion signals in different directions. A natural question to ask is why there should be an opponent stage in the motion pathway in the first place, since it is certainly not just for making locally balanced stimuli (such as the paired dot patterns) nontransparent. One possibility is that suppression may help to enhance the directional selectivity of neurons (Reichardt, 1961; Barlow and Levick, 1965). For example, subtractive suppression between two units tuned to opposite directions of motion can remove the motion-independent portion of the responses and therefore increase cells' degrees of directional selectivity. To test this hy-

\section{MT Cell}
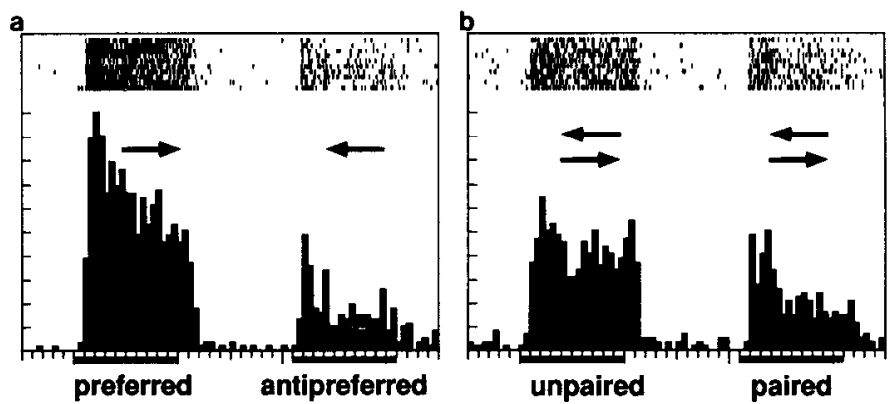

Figure 6. An MT cell tested on the paired dot patterns with 1 pixel vertical offset between the two dots in each pair. $a$, Responses to single sets of random dots moving in its preferred and the antipreferred directions. $b$, Responses to the paired and the unpaired dot patterns with one set of dots moving in the preferred direction and the other the antipreferred direction. One small vertical division represents 12.4 spikes/ sec. 


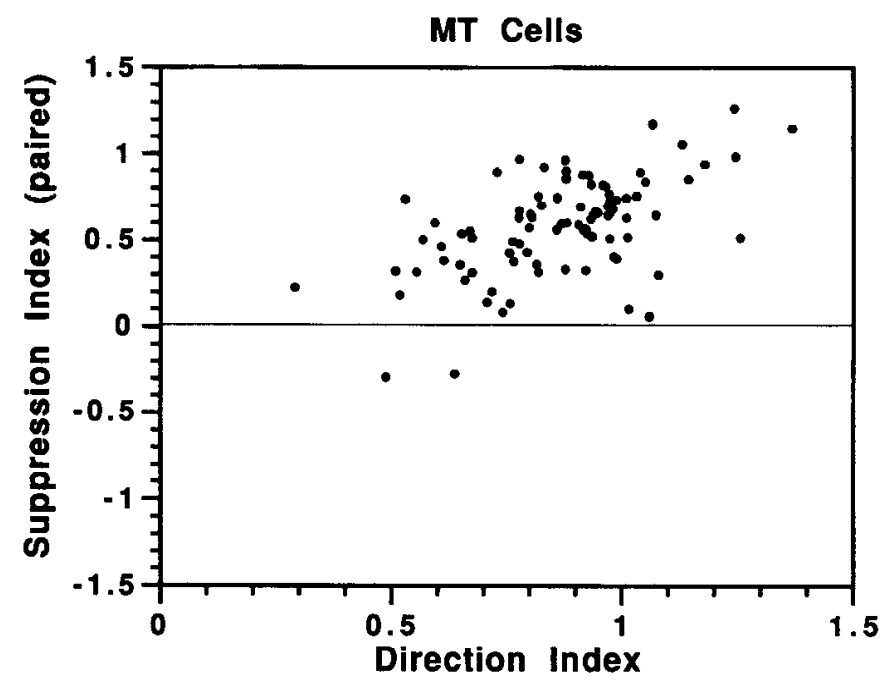

Figure 7. The suppression indices of MT cells, measured with the paired dot patterns, plotted against their directional indices.

pothesis, we plot the directional index of each cell against its suppression index measured with the paired dot patterns in Figure 7. The directional index is defined as one minus the ratio of a cell's antipreferred response to its preferred response (again, the spontaneous rate was subtracted before calculation):

$$
D I=1-\frac{\text { antipreferred response }}{\text { preferred response }} .
$$

A large index value means that the cell is highly directionally selective. There is indeed a positive correlation between the two indices (Spearman rank correlation $\rho-0.51, p=0.0001$ ). $\Lambda$ weaker correlation exists when the unpaired suppression index is used (Spearman rank correlation $\rho=0.26, p=0.015$ ). That the correlation is stronger under the paired condition reflects the fact that the paired dot patterns are better probes for cells' local suppressive mechanisms.

Another, and perhaps more important, functional role of directional suppression could be motion noise reduction. The spatially and temporally uncorrelated component in a stimulus is noise to the motion system. Any changes in stimulus light intensity distribution that are not generated by coherent motion contribute to such noise. For example, motion noise is generated when the light in a room is turned on or off. Another example would be sudden appearance or disappearance of an object or part of an object, which emits or reflects light. This situation is quite common in a natural environment, such as forest, where there are many small objects (leaves, branches, etc.) with different light reflectances and spatial orientations. When these objects undergo coherent motion (e.g., under the influence of wind), they also generate flicker noise due to the random changes of their orientations, and random occurrence of occlusions and the removals of occlusions. Such spatially and temporally uncorrelated noise has a relatively uniform spatiotemporal Fourier spectrum and, from the point of view of motion energy detectors (Adelson and Bergen, 1985; Watson and Ahumada, 1985), it contains equal amounts of "motion" signals in all directions. By introducing suppression among detectors tuned to different directions of motion, the responses of these detectors to noise can be greatly reduced.

We tested this hypothesis by recording from MT cells using noise patterns made of flickering random dots. We used two types of noise patterns, one derived from the paired dot patterns and the other from the unpaired dot patterns. They were identical to the moving patterns in all aspects except that none of the dots moved from frame to frame. Each dot stayed in a fixed random location during its lifetime ( 7 frames or $117 \mathrm{msec}$ ) and then was replotted at a new location. If the directional suppression observed in MT is important for noise reduction, then cells with stronger suppression should respond less to the noise patterns. An example of an M'T cell's responses to the noise patterns is shown in Figure 8, together with its preferred, antipreferred, paircd, and unpaircd responscs. The responses to both the paired and the unpaired dot patterns are much weaker than its preferred direction response, indicating very strong directional inhibition for this cell. Its noise responses are also quite small, as expected.

To quantify the population result we introduce two noise indices for each cell, one for the paired noise and the other for the unpaired noise. They are the noise responses expressed as percentages of the preferred direction response:

\section{MT Cell}
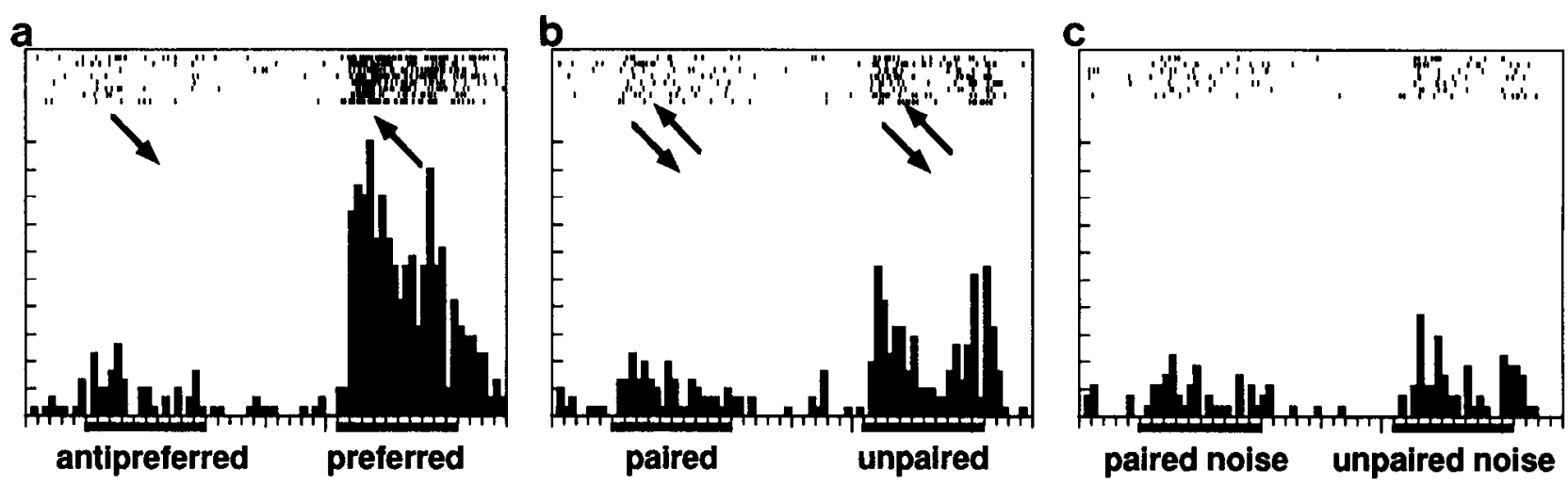

Figure 8. Noise response of an MT cell. $a$, Responses to single sets of random dots moving in its preferred and the antipreferred directions. $b$, Responses to the paired and the unpaired dot patterns with one set of dots moving in the preferred direction and the other the antipreferred direction. $c$, Responses to the paired and the unpaired noise patterns of flickering dots. One small vertical division represents 7.8 spikes/sec. 


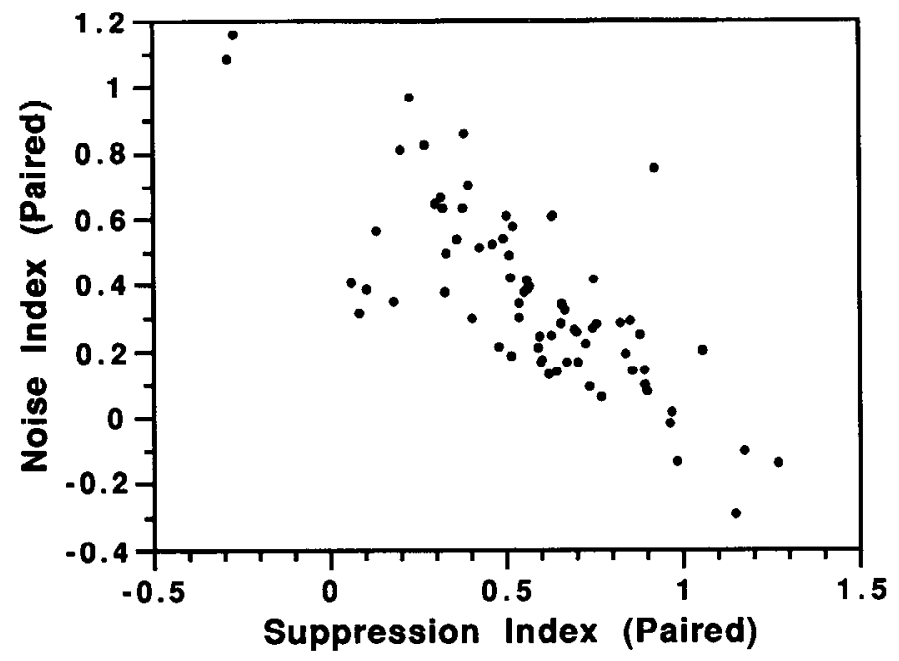

B

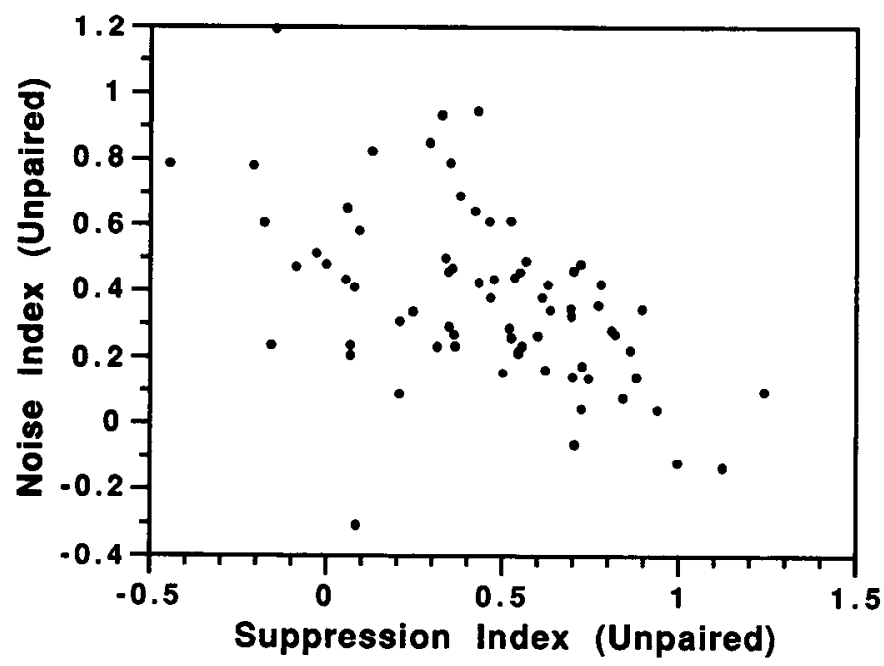

Figure 9. Noise index versus suppression index for MT cells. $a$, The paired case. $b$, The unpaired case.

$$
\begin{aligned}
N I_{\mathrm{p}} & =\frac{\text { paired noise response }}{\text { preferred response }} \\
N I_{\mathrm{up}} & =\frac{\text { unpaired noise response }}{\text { preferred response }}
\end{aligned}
$$

The background rate was again subtracted before calculation. A large index indicates a strong noise response. We plotted the noise index against the suppression index for both the paired and the unpaired cases. The results, shown in Figure 9, agree with our prediction quite well. There is a strong and negative correlation (Spearman rank correlation $\rho=-0.75, p=0.0001$ ) between a cell's response to the paired noise pattern and the degree of directional suppression measured with the paired dot pattern (Fig. 9a). The correlation is weaker but still highly significant (Spearman rank correlation $\rho=-0.52, p=0.0001$ ) for the unpaircd casc (Fig. $9 b$ ). The main difference between Figure $9, a$ and $b$, is that points in the latter figure are more spread out for the less suppressive indexes. Again, the correlation is stron-

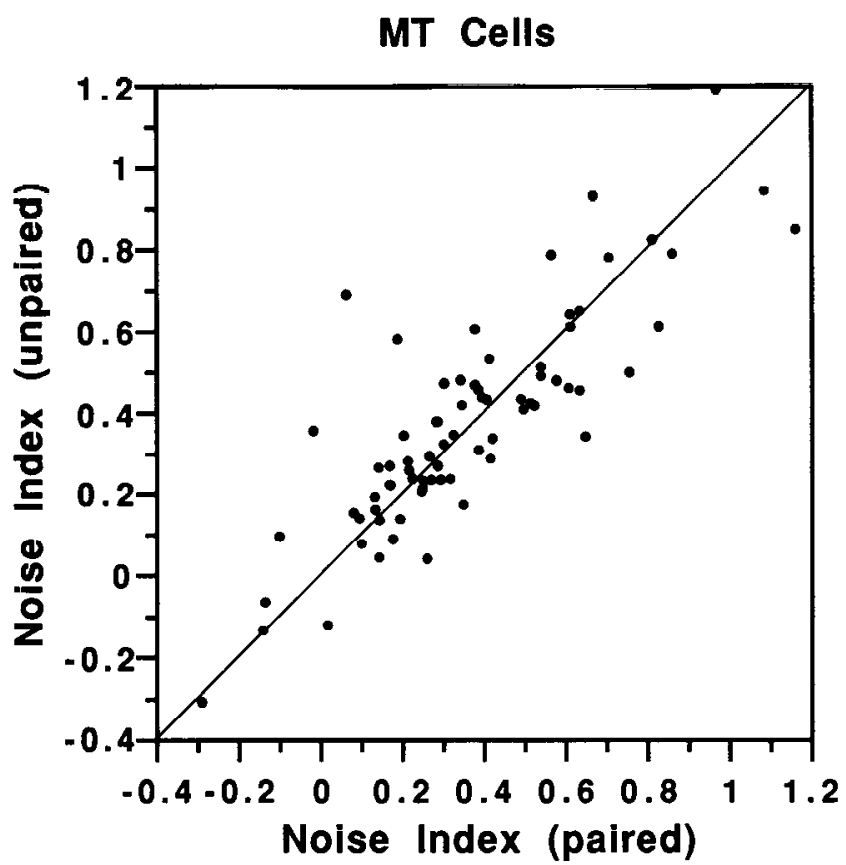

Figure 10. The noise index of MT cells for the paired flicker is plotted against that for the unpaired flicker.

ger under the paired condition, reflecting the fact that the paired dot pattcrns arc better probes for the cells' local suppressive mechanisms. It is also worth pointing out that these correlations are stronger than those between suppression and directionality (Fig. 7).

The cells' responses to the two types of noise patterns are essentially the same. This is demonstrated by plotting the cells' responses to the paired noise against that to the unpaired noise patterns in Figure 10. The two responses are highly correlated and not significantly different from each other. This observation serves as an extra control for our results on the paired and the unpaired moving dot patterns presented in Figure 4. It indicates that the better response (or weaker suppression) of MT cells to the unpaired dot patterns than to the paired ones is not due to the positional effects of pairing and unpairing of dots. The motion of the dots is essential.

\section{Average $M T$ responses to different stimuli}

We have shown that a good fraction of MT cells responded significantly stronger to the unpaired dot patterns than to the paired dot patterns. These cells could form the physiological basis of the perceptual difference of these patterns. A hidden assumption here is that the brain could selectively "listen" to this subpopulation of cells in MT. We wondered if one could still tell the two types of patterns apart based on the average activity of all MT cells. We calculated the average responses of all recorded $\mathrm{MT}$ cells to five different types of stimuli: single sets of dots moving in the preferred and the antipreferred directions, the paired and the unpaired dot patterns with one set of dots in the preferred and the other antipreferred directions, and the unpaired noise pattern. We did not include the responses to the paired noise pattern since they are similar to the unpaired noise pattern (see Fig. 10). The background firing rates of all cells are subtracted before calculation. The responses of cells to different stimuli are first normalized by their preferred direction responses before averaging. The results are shown in Figure $11 a$. 
A

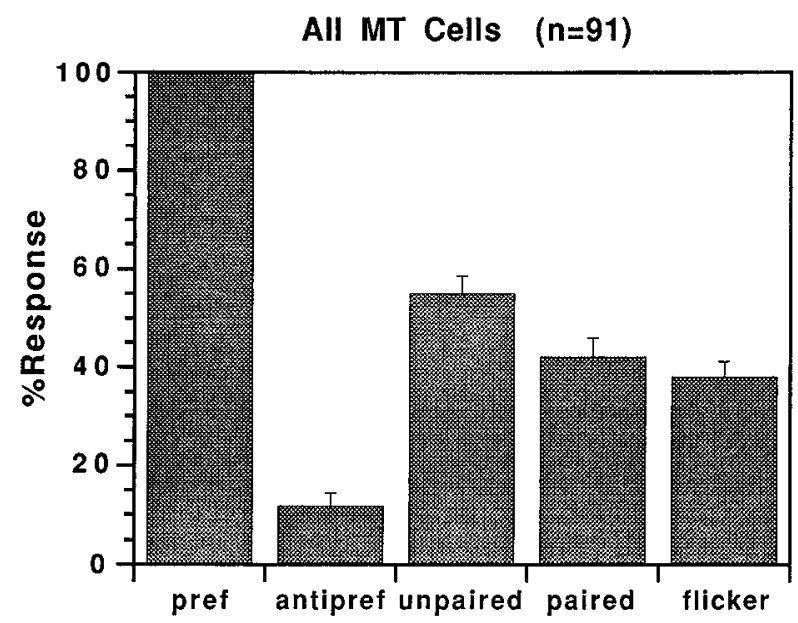

C

MT Cells, Paired $=$ Unpaired (46/91)

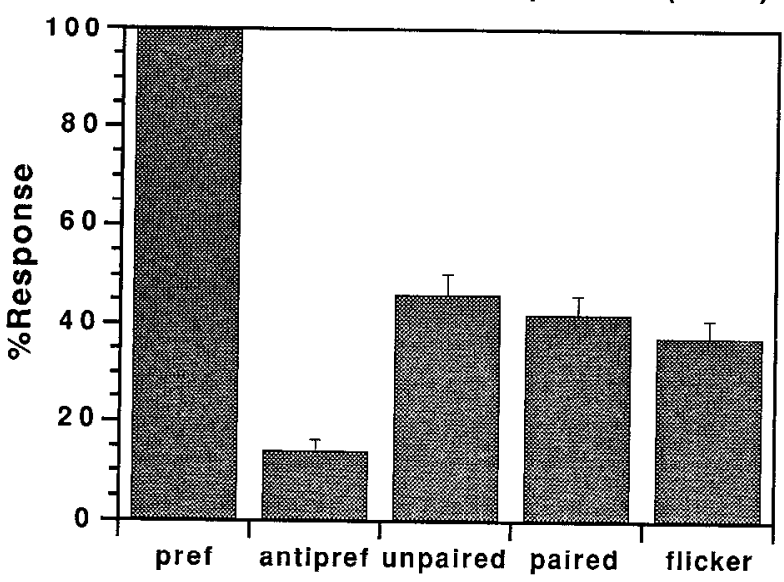

$\mathbf{B}$

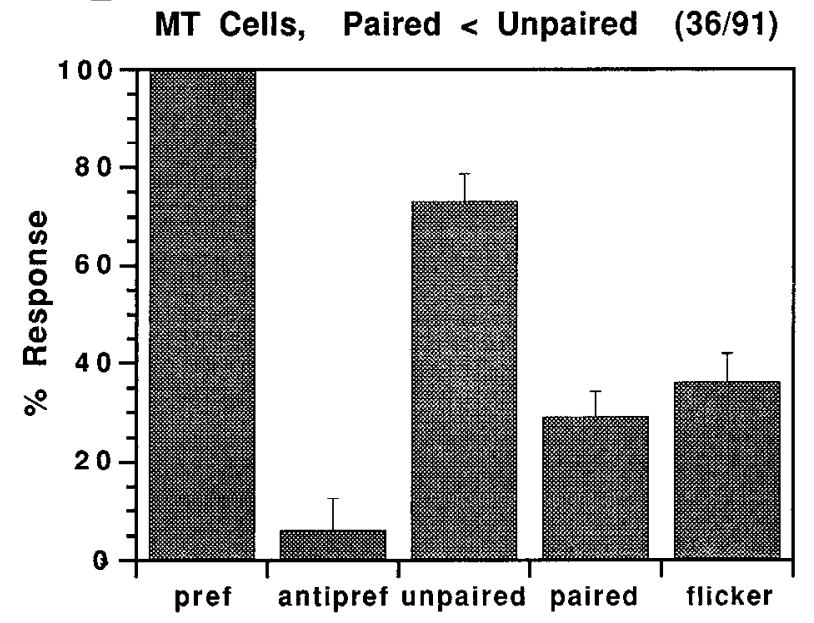

D

MT Cells, Paired > Unpaired (9/91)

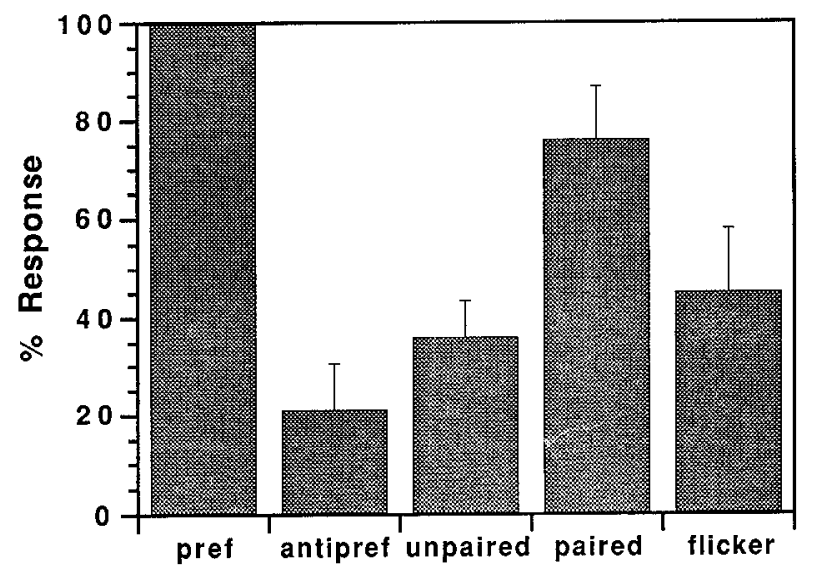

Figure 11. The average responses of MT cells to different stimuli. The average was taken over all recorded MT cells (a), MT cells that responded better to the unpaired dot patterns than to the paired dot patterns $(b)$, MT cells that responded equally well to the two types of patterns $(c)$, and MT cells that responded better to the paired dot patterns than to the unpaired dot patterns $(d)$. The error bars represent SEs.

The error bars represent SEs. It is clear from this figure that on the average, MT cells are suppressed by $45 \%$ from the preferred direction response when the unpaired moving dot patterns are used. The average suppression for the paired moving dot patterns, on the other hand, is about $60 \%$. The difference between the two types of patterns is statistically significant (two-tailed paired $t$ test $p=0.0002$, Wilcoxon signed-rank test $p=0.0001$ ). This indicates that MT cells as a population could still distinguish the two types of displays with different perceptual transparency. Furthermore, the average response to the paired dot patterns is not significantly different from the average noise response (two-tailed paired $t$ test $p=0.086$, Wilcoxon signedrank test $p=0.083$ ). This is also reflected in the responses of many individual cells (Fig. 8 gives an example). The result agrees with the perceptual phenomenon that the paired dot patterns look rather like the noise pattern even though physically they are generated in quite different ways.

We also calculated the average responses of the cells in the three different categories separately. They contain the cells whose responses to the unpaired dot patterns are significantly stronger than, not significantly different from, and significantly weaker than that to the paired dot patterns, respectively. The results are shown in Figure $11 b-d$. These figures reveal several other interesting differences among the cells in the three categories. First, the cells that responded better to the transparent patterns than to the nontransparent ones are most directional while those with the opposite behavior are least directional among the three groups. This is indicated by the normalized average responses to the antipreferred motion (als $\Theta$ compare the directionality of the cells in Figs. 1-3). Second, the cells that respond better to the paired than the unpaired dot patterns generate the largest average noise response. These results together indicate that the cells that respond better to the nontransparent patterns than to the transparent ones are less selective to motion. Finally, for the cells that responded equally well to the two types of patterns, the average response to the unpaired dot patterns, as well as that to the paired dot patterns, is not significantly different from the average noise responses. This result is also reflected in the responses of many individual cells (see Fig. 12 for an example). If we assume that the noise response level is the baseline where paired or unpaired dot response can be suppressed to, the result provides a possible explanation for the fact that these cell cannot 


\section{MT Cell}
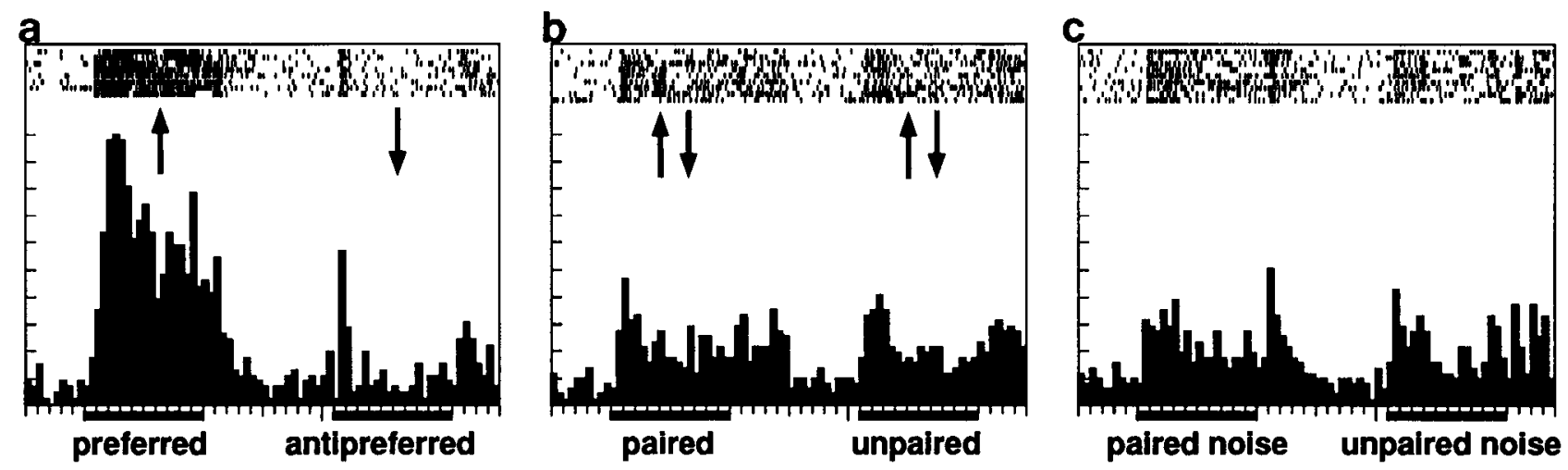

Figure 12. Noise response of an MT cell. $a$, Responses to single sets of random dots moving in its preferred and the antipreferred directions. $b$, Responses to the paired and the unpaired dot patterns with one set of dots moving in the preferred direction and the other the antipreferred direction. $c$, Responses to the paired and the unpaired noise patterns. One small vertical division represents 11.5 spikes/sec.

distinguish the two types of displays: the suppression is already maximum for the unpaired dot patterns, and there is perhaps no room left for further suppression when the paired dot patterns are used.

\section{$\checkmark 1$ responses}

We also recorded from V1 cells using the same set of stimuli. The purpose of this study is to find out which area better correlates with the perceptual differences of the stimuli. We recorded a total of $110 \mathrm{~V} 1$ cells. Among them, 36 cells werc directionally selective. The responses of a representative directionally selective V1 cell to the paired and the unpaired dot patterns are shown in Figure 13, together with its preferred and the antipreferred responses. The cell's responses to the paired and the unpaired dot patterns are somewhat smaller than its preferred direction response, indicating a weak suppression between the preferred and the antipreferred directions. The responses to the paired and the unpaired dot patterns are not significantly different from each other. This is the typical situation for most V1 cells we recorded.

As in our MT analysis, we computed for each directional V1 cell the suppression indices for the paired and the unpaired dot patterns. Non-direction-selective cells were not included in the analysis. Similar to Figure 4 , we plot in Figure 14 for each V1 cell the paired suppression index against the unpaired suppression index. This figure shows that the majority of the directional $\mathrm{V} 1$ cells showed some suppression between the preferred and the antipreferred directions, although it is much weaker than that found in MT. There are more cells below the diagonal line than above it but the bias is much smaller in comparison with MT (see Fig. 4). To examine the behavior of individual cells, we performed the two-tailed $t$ test for each cell to see if its mean responses to the transparent and the nontransparent patterns are significantly different from each other. The cells that did show significant difference are shown as solid dots in Figure 14 and the rest as open dots. Seventy-five percent (27 of 36) of the directional VI cells could not tell the two types of patterns apart. Only $17 \%$ (6 of 36 ) responded significantly stronger to the unpaired dot patterns than to the paired ones. This fraction is $40 \%$ for MT cells. Finally, $8 \%$ ( 3 of 36 ) of cells showed the opposite behavior.

The difference in the degrees of the directional suppression between V1 and MT is correlated with the difference in their directionality. To see this, we have plotted in Figure 15 the paired suppression index against the direction index for $\mathrm{V} 1$ cells. By comparing this figure with the similar one for MT cells (Fig. 7), we see that the two distributions form a continuum and that very directional V1 cells are not different from typical MT cells. We conclude that the difference between V1 and MT is only quantitative. Like MT cells, there is also a significant correlation between the paired suppression index and the directional index (Spearman rank correlation $\rho=0.34, p=0.045$ ). The correlation is stronger when the unpaired suppression index is plotted against the directional index (Spearman rank correlation $\rho=0.61, p=$ 0.0003 ). A previous study from our laboratory failed to find such correlation (Snowden et al., 1991) although the trend was present for cells with high directional indexes. This difference is probably caused by the fact that the directional indexes were higher overall for the V1 population in the present study.

We also recorded noise responses of V1 cells. In Figure 16,

\section{V1 Cell}
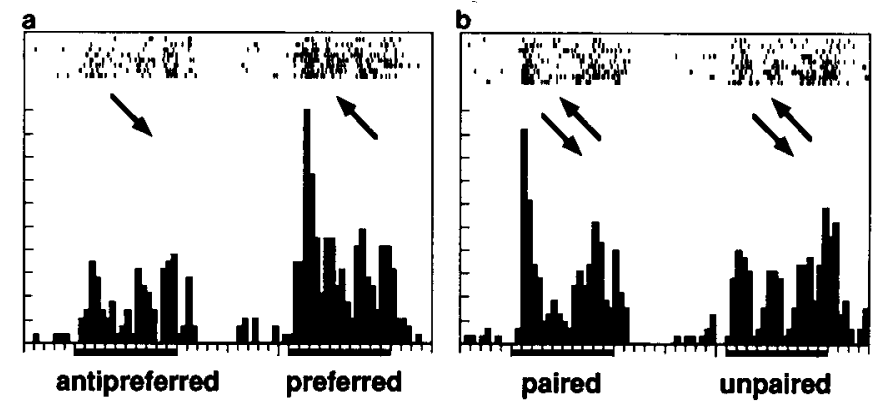

Figure 13. A V1 cell with equal responses to the paired and the unpaired dot pattern. See Figure 1 for details. One small vertical division represents 7.3 spikes/sec. 


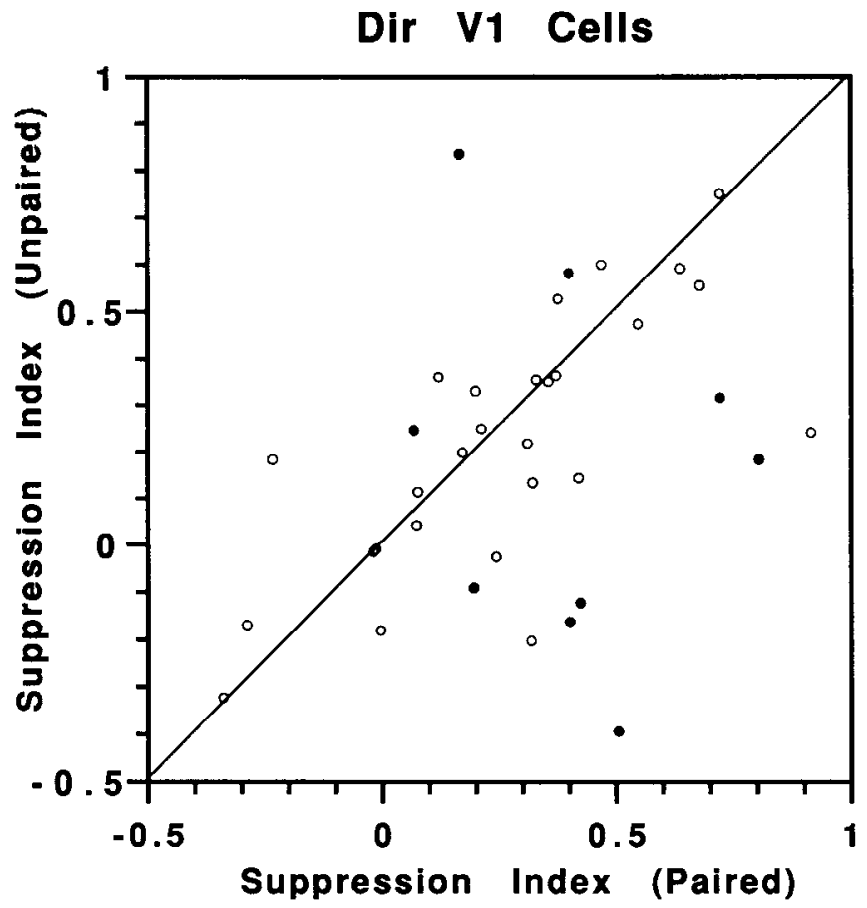

Figure 14. For each V1 cell, the suppression index for the paired dot pattern is plotted against its suppression index for the unpaired dot pattern. The cells with significantly different responses to the two types of patterns based on a two-tailed $t$ test are shown as solid circles. The rest are shown as open circles.

$a$ and $b$ are the plots of the noise index against the suppression index for the paired and the unpaired cases, respectively. The two indices appear to be negatively correlated, but, unlike MT cells, the correlations are not significant (Spearman rank correlation $\rho=0.29, p=0.089$ for the paired case, and $\rho=0.31$, $p=0.066$ for the unpaired case). The average noise response of $\mathrm{V} 1$ cells is about $63 \%$ of the preferred direction response, much higher than the MT noise response, which is only $37 \%$ of the preferred response. Since V1 cells have much weaker directional suppression, this result is consistent with our proposal that one of the functions of directional suppression is noise reduction in motion processing.

The average responses of all directional V1 cells we recorded to different types of stimuli are shown in Figure 17. Unlike for MT cells (see Fig. 11a), the average V1 response to the paired dot patterns is not significantly different from that to the unpaired dot patterns (two-tailed paired $t$ test $p=0.081$, Wilcoxon signed-rank test $p=0.19$ ). This means that an observer could not reliably tell the two types of displays apart by "listening" to the overall responses of all directional V1 cells. Another clear difference between $\mathrm{V} 1$ and $\mathrm{MT}$ is that the average responses of directional V1 cells to the paired and the unpaired dot patterns as well as to the noise patterns, expressed as percentages of the preferred direction responses, are all higher than those of MT cells. All these observations are consistent with the fact that the amount of suppression between the preferred and the antipreferred directions is much weaker in Vl.

\section{Issues related to spatial scale difference between $V 1$ and $M T$}

In our V1 and MT experiments reported above, we did not first search for the optimal stimulus parameters (such as display size, dot density, and speed, etc.) for each isolated cell and then record

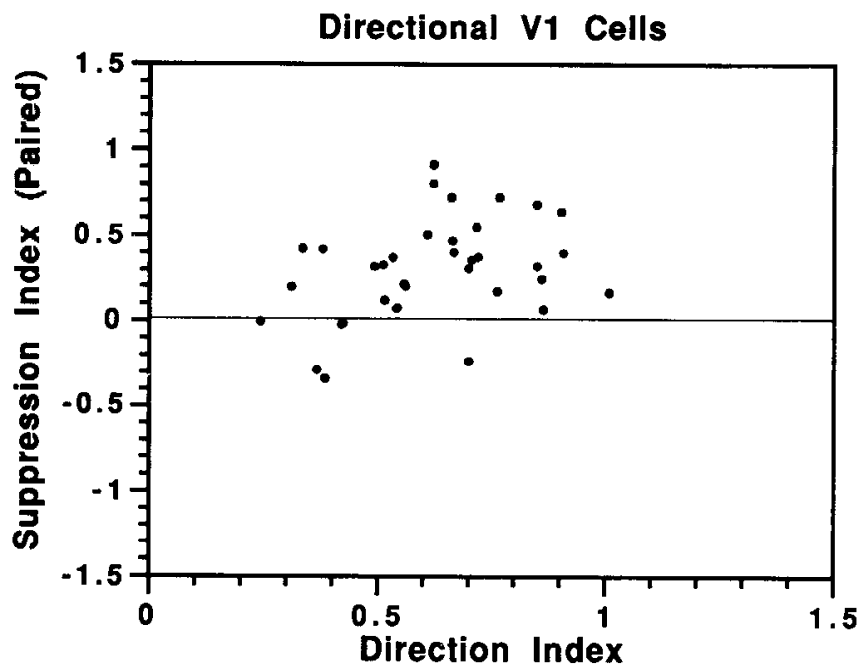

Figure 15. The suppression indices of V1 cells, measured with the paired dot patterns, plotted against their directional indices.

under these parameters. We think that this approach is justified because here we are not interested in optimal tuning properties of the cells. Instead, our goal was to find out how a population of V1 or MT cells responds to two fixed types of stimuli, one of which is perceptually transparent and the other nontransparent. This approach allows us to study neural population coding of a well-defined perceptual attribute. In fact, the degree of perceptual transparency of a stimulus depends on its parameters. Had we changed the stimulus parameters markedly from cell to cell, the results would be more difficult to interpret.

However, V1 and MT cells are known to differ in a major way: the receptive field sizes of the former are much smaller than those of the latter. When responsive properties of the cells in the two areas are being compared, it does not seem fair to use stimuli of the same size and dot density. If we try to rectify the problem by simply scaling these parameters according to the receptive field sizes of the cells, then the stimuli used for V1 and MT will differ systematically in their degrees of perceptual transparency. This again will make the comparison between the two visual areas difficult.

The scale difference between $\mathrm{V} 1$ and MT receptive ficlds turns out not to be a major issue for the present study because previous work from our laboratory indicates that responses of V1 cells are not very sensitive to dot density and stimulus size in the range used in the present experiments (Snowden et al., 1991). Dot densities from 0.45 to $28.8 \mathrm{dots} / \mathrm{degree}^{2}$ were used in each surface (a 64 -fold change in density) with no systematic change in suppression. In further experiments, $24 \mathrm{~V} 1$ cells were tested with two types of transparent stimuli, one containing 64 dots per surface and the other 32 dots per surface. No systematic difference was seen with the two densities. Similar results were found for $15 \mathrm{MT}$ neurons tested in the same manner. Thus, dot density, varied in the range used in the present experiments, does not systematically effect the suppression index of V1 cells. We also used different-diameter stimuli in V1 and found no significant influence of stimulus size on suppression index (Snowden et al., 1991). Therefore, the fact that the standard stimuli used in this study were confined in the classical receptive fields of MT cells but were much larger than the receptive fields of V1 cells (see Material and Methods) is unlikely to affect our results. 
A

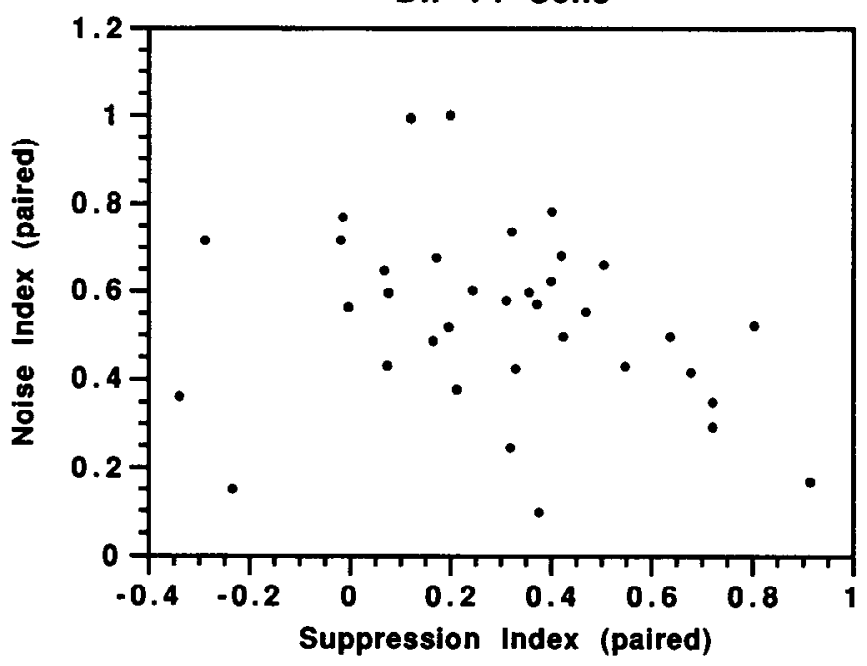

B

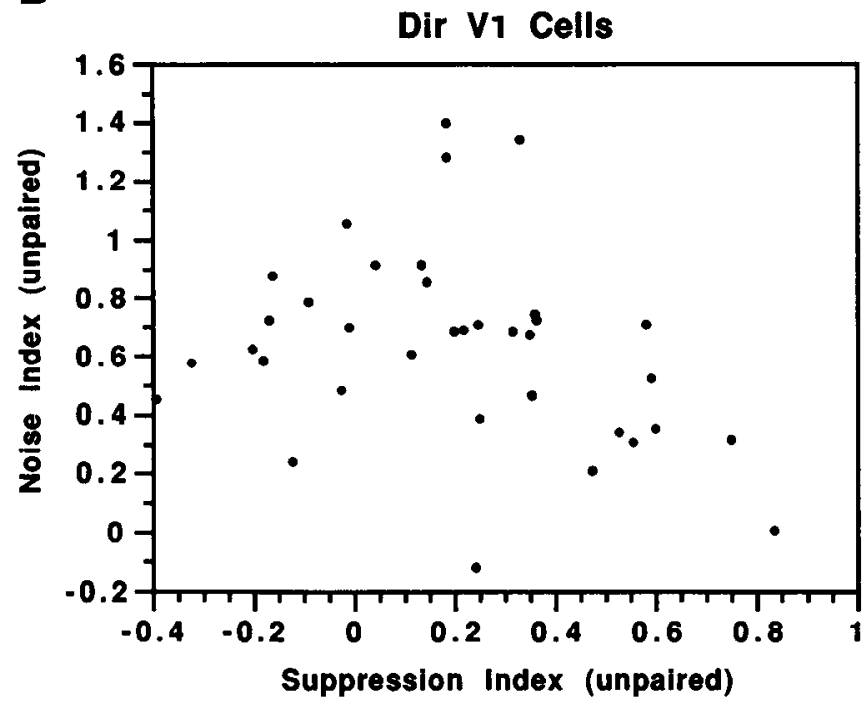

Figure 16. Noise index versus suppression index for V1 cells. $a$, The paired case. $b$, The unpaired case.

We nevertheless did a control experiment in the present study in order to readdress this issue using paired and unpaired dot patterns. We recorded from V1 cells using a set of stimuli with radius equal to $1.5^{\circ}$ while keeping all the other parameters identical to the standard set of stimuli. The stimulus area was therefore four times smaller and the dot density four times higher than the standard case. For 23 V1 cells recorded this way, seven were found to be directionally selective. Among the seven cells, five showed no significant differences between their responses to the paired and the unpaired dot patterns, one responded significantly better to the paired than to the unpaired dot pattern and the remaining one cell showed the oppositc bchavior. These results are consistent with the data shown in Figure 14 under the standard condition. We believe part of the reason for the lack of dot density dependence is that all dots in our stimuli had limited lifetime. Each dot was replotted more than eight times to new randomly chosen locations during the 1 sec display period (see Materials and Methods). The dots were thus much
All Dir V1 Cells $(n=36)$

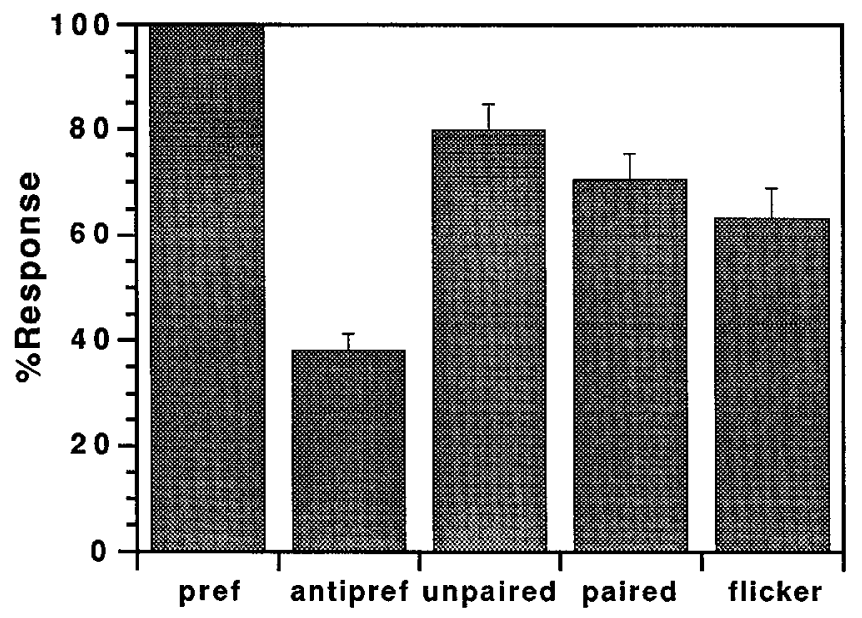

Figure 17. The average responses to different stimuli over all directional V1 cells. Responses are expressed as percentages of the preferred direction responses.

more uniformly distributed than would otherwise be the case if they were not repeatedly replotted.

Note that in the above control experiment we did not scale the stimulus size and dot density based on the actual scale differences between the V1 and MT cells we sampled. Since the receptive field area of MT cells we recorded was more than 100 times larger than the V1 cells, a proportionate scaling down of the stimulus area would generate paired and unpaired dot patterns that are so dense that both will appear nontransparent. These two types of patterns would certainly generate no differences in the responses of $\mathrm{V} 1$ cells. The result, however, would add nothing to the issue of whether V1 could distinguish transparent and nontransparent motions because at this high density there is little perceptual difference between the patterns. We therefore did not perform such an experiment.

\section{$M S T$ responses to the paired and the unpaired dot patterns}

During the course of recording from MT, we also recorded seven cells from area MST. They were considered as MST cells based on their relative positions to MT cells we recorded (a few millimeters more anterior), their large receptive fields that included the fovea and the ipsilateral visual space, and their positionally invariant spiral tuning (Graziano et al., 1993). Similar to Figures 4 and 14, we plot the cells' suppression indices for the paired and the unpaired dot patterns against each other in Figure 18. Interestingly, all seven cells fall below the diagonal line. Five of them responded significantly stronger to the unpaired dot patterns than to the paired ones. Although seven cells is too small a population to draw a conclusion from, this, together with our V1 and MT results, does suggest that along the motion pathway, there is a progressive increase of the proportion of cells that could distinguish the transparent patterns from the nontransparent ones.

\section{Discussion}

We have been investigating the problem of motion transparency in our laboratory using a combination of psychophysical, physiological, and computational approaches. As reported in the preceding companion article, our psychophysical experiments with the paired dot patterns demonstrate that when a display 


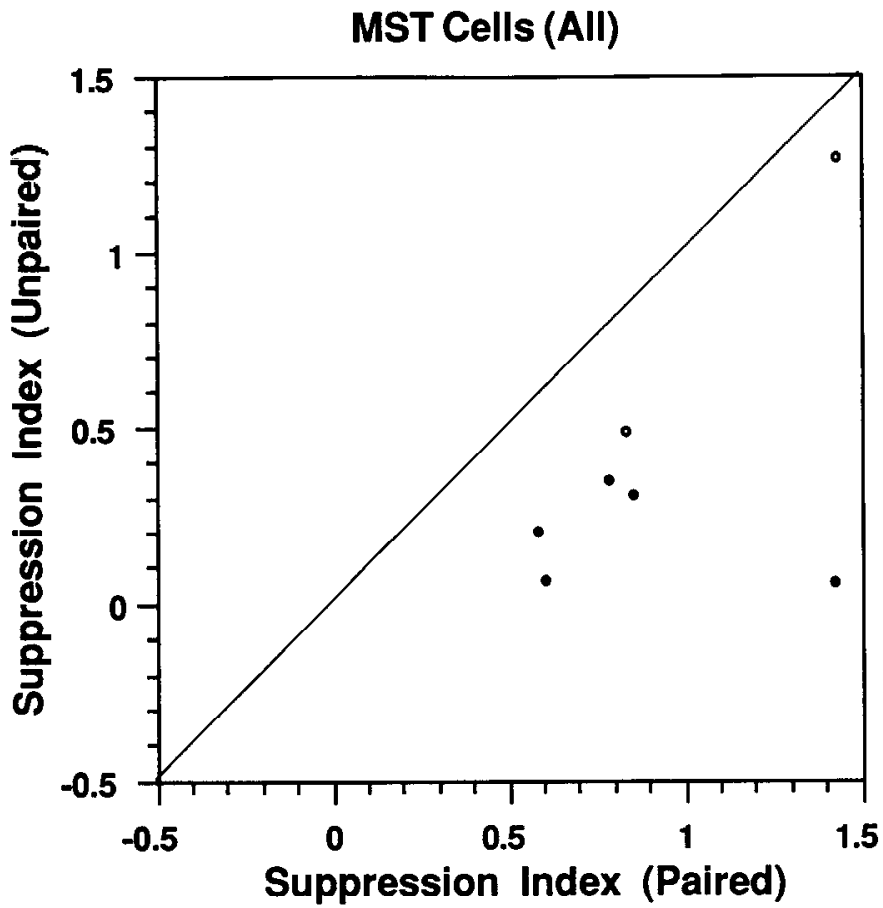

Figure 18. For each MST cell, the suppression index for the paired dot pattern is plotted against its suppression index for the unpaired dot pattern. The cells with significantly different responses to the two types of patterns based on a two-tailed $t$ test are shown as solid circles. The rest are shown as open circles.

contains two opposing motion vectors in every location (and thus is locally well balanced) it actually looks like flicker instead of two transparent moving surfaces (Qian et al., 1994a). Transparency accurs only when there are locally unbalanced motion signals in different directions, as in the unpaired dot patterns. Our computational studies, reported in the following companion article, indicate that these observations can be explained by proposing an opponent or suppression stage in the motion pathway, at which motion signals from different directions locally inhibit each other (Qian et al., 1994b). Such an opponent stage could tell transparent and nontransparent displays apart by measuring the locally unbalanced motion signals in these displays. In this article we showed that the behavior of MT cells is consistent with the opponent stage we predicted. More specifically, we found that MT cells show strong suppression among different directions of motion, and that the suppression is significantly stronger for the paired dot patterns than for the unpaired ones. The responses to the paired dot patterns arc in fact not significantly different from the responses to flicker noise patterns. These results correlate well with our perception that the unpaired dot patterns are perceptually transparent while the paired ones look more like flicker. They provide evidence that MT could be the cortical locus for the perception of transparency. Similar proposal has been made by Stoner and Albright (1991).

We also recorded from V1 cells using the same set of stimuli. We found that like MT cells, directional V1 cells also show suppression between their preferred and the antipreferred directions of motion. The degree of suppression, however, is much weaker. The proportion of directional V1 cells that responded differently to the paired and the unpaired dot patterns is also much smaller. This is consistent with the assumption that di- rectional suppression is the mechanism for distinguishing transparent and nontransparent displays. Unlike MT cells, the average responses of all directional $\mathrm{V} I$ cells to the paired and the unpaired dot patterns are not significantly different from each other. We conclude that MT activity correlates better with our perception of motion transparency than V1 does. Of course, a correlation between neural activity in MT and transparent motion perception does not establish a causal relationship. It remains possible that MT cells merely reflect directional suppression originatcd in a different area. Further research is required to resolve this issue. In addition, since we did find a small fraction of Vl cells that showed strong suppression and responded significantly better to the unpaired dot patterns than to the paired ones, we cannot completely rule out the possibility that V1 may also contribute to transparent motion perception. Although the fraction of directional V1 cells that could tell the two types of patterns apart is much smaller than MT, the absolute number could be greater since V1 is bigger than MT by a factor of 20. An implicit assumption behind this possibility, however, is that the brain could somehow selectively listen to a very small fraction of "privileged" V1 cells while ignoring the overwhelming activities from the rest. If, instead, the brain had to use the average activities of all cells in an area (or a small region within an area), then, based on Figure 17, V1 could not be the physiological basis of transparent motion perception.

In the paired dot patterns, there are always precisely two motion vectors in a small area of about $0.4^{\circ}$ (the maximum separation between the two dots in each pair). The fact that the average response of MT cells to the paired dot patterns is not significantly different from the average response to the flicker noise patterns suggests that MT could not detect more than one motion, in the absence of other cues (see below). On the other hand, the average MT response to the unpaired dot patterns is significantly above the level of the noise response. Since the physical difference between the paired and the unpaired dot patterns occurs at the scale of about $0.4^{\circ}$, these displays can only be distinguished with units of similar sizes. The receptive fields of the MT cells we recorded were relatively large $\left(6-10^{\circ}\right)$, and they contained the whole paired or unpaired patterns within them. For MT cells to be able to distinguish the paired and the unpaired dot patterns, we have to assume that the receptive field of an MT cell is composed of many small subunits of size about $0.4^{\circ}$, and that directional suppression is performed within each subunit. If we further assume that the response of an MT cell is determined by the sum of the thresholded outputs of all its subunits, the cell will then respond to the two types of dot patterns differently (see Fig. 8 of the preceding companion article, Qian et al., 1994a).

Since the suppression among different directions of motion is strong in MT, we speculated on its possible functions. One obvious possibility is that it enhances the directionality of the cells. This view is supported by our finding that the degrees of suppression of both MT and V1 cells are correlated with their directionality, and that MT cells show stronger suppression and at the same time higher degrees of directionality. From the computational point of view, however, the high directionality can be achieved without introducing explicit suppression among units tuned to different directions of motion. In fact, a linear receptive field (Adelson and Bergen, 1985; Watson and Ahumada, 1985) followed by a threshold or squaring nonlinearity can produce units with directional indices near 1 . The presence of strong suppression in MT is particularly puzzling since it has 
the seemingly undesirable effect of preventing the visual system from representing more than one motion at a given spatial location. We therefore looked for other explanations. Our recordings with the noise patterns suggest an important alternative. The striking correlation between the noise response and the degree of suppression shown in Figure 9 suggests that a major function of directional suppression could be noise reduction in the motion pathway. As we mention before, there is strong spatially and temporally uncorrelated noise in the natural environment. It thus appears important for the motion system to distinguish true motion signals from the spatiotemporal energies generated by noisy components in the stimuli.

It should be pointed out that the term "noise response" used in our study should not be identified with the intrinsic noise within the nervous system. The former is defined as the response of a cell to an external flicker stimulus (considered as noise input to the motion system) while the latter is charactcrized by the variance of a cell's response (to any stimulus) over repeated trials or the variance of the interspike interval of a response. In fact, while we report here that the noise response of MT cells is much smaller than that of V1 cells, the variabilities of V1 and MT responses have been found to be similar (Snowden et al., 1992; Softky and Koch, 1993). We suggested that the weaker noise response in MT is due to the stronger suppression in that area. The equal variability of V1 and MT activities indicates that the strong suppression in MT does not help to reduce the intrinsic noise within the system. This is expected if the intrinsic noises from different V1 cells projecting to an MT cell are not strongly correlated.

Our recordings with flicker noise patterns also prompt us to ask what portion of a cell's response to a moving pattern is truly involved in coding motion. The standard answer is all of the response except the amount equal to the background firing ratc recorded with a blank screen. According to this view, one would conclude, based on Figure $11 a$, that on the average, MT cells detect significant amount of motion signals in the paired dot patterns, since the cells' average paired response is significantly above the background rate, which is set to $0 \%$ in that figure. We think this interpretation is incorrect because the difference between a moving pattern and a blank screen is not just motion. The mere presence of something in one case but not in the other must account for a significant part of the difference in the responses. The best control display for determining the motionindependent part of a response to a moving pattern should be the one that is identical in all aspects to the moving pattern but lacking the coherent motion. From the point of view of motion energy models (Adelson and Bergen, 1985; Watson and Ahumada, 1985), the control display should contain equal amount of spatial and (separately) temporal frequency contents as the moving pattern, but without the spatiotemporal structure in the moving pattern. The flicker noise patterns we generated as controls for the paired and the unpaired dot patterns satisfy this criterion. We therefore compared the average paired dot response with the average noise response in Figure $11 a$. Since the two responses are not significantly different from each other, we concluded that the average MT activity does not carry motion information for the paired dot patterns.

Fifty percent of the MT cells we recorded from showed no significant difference in their responses to the paired and the unpaired dot patterns. These cells displayed particularly strong suppression between opposite directions of motion, in comparison with the preferred direction responses. In fact, the responses of these cells to the paired and the unpaired dot patterns were suppressed so much that they both were not significantly different from the responses to the flicker noise patterns. This result suggests why the suppressive mechanism did not help these cells to distinguish the two types of patterns: their responses to the unpaired dot patterns were already maximally suppressed to the noise level and there is no room left when we try to increase the suppression further by using the paired dot patterns.

Although only $40 \%$ of our MT cells responded significantly better to the transparent patterns than to the nontransparent ones, the difference they created was strong enough to generate a significant difference in the overall responses to the two types of patterns, averaged over all MT cells we recorded, as shown in Figure $11 a$. Through comparison with the average noise response, Figure $11 a$ also indicates that on the average, $37 \%$ of an MT cell's response to a single set of dots moving in its preferred direction is unrelated to motion, while the remaining $63 \%$ carries motion information. For the unpaired dot patterns, the strength of the motion signal (the part above the flicker noise level) is still significant but reduced to $29 \%$ of the preferred response carrying the motion signal, due to the suppression between opposite directions of motion. Transparent motion should therefore be much harder to detect than single motion. This conclusion is supported by the psychophysical observations that the upper displacement limit is smaller under the transparent condition (Snowden, 1989). Finally, when the paired dot patterns were used to maximize the inhibition between opposite directions of motion, the response was suppressed so much that it is no longer significantly different from noise response. This result is consistent with the observation that the paired dot patterns do not appear to contain coherent motion. In fact, they look rather like flicker noise even though physically, the paircd dot patterns are quite different from the flicker patterns.

We show in this article that when the pairing process is applied to a transparent random dot display, the resulting paired dot pattern generates an increased suppression in MT between opposite directions of motion. We argued that this is the case because motion signals in opposite directions in a paired dot pattern are better balanced locally and are thus more likely to cancel each other within the subunits of MT receptive fields. This explanation predicts that if the degree of balance between opposite directions of motion in a transparent random dot pattern is decreased, the strength of motion suppression should also decrease. This is indeed what we observed in a previous study from our laboratory (Snowden et al., 1991; see also Britten and Newsome, 1990). In that study, displays were divided into either two or six adjacent but nonoverlapping bands. Dots in every other band moved in one direction and those in the remaining half of the bands moved in the opposite direction. Individual MT cells were recorded using these less balanced patterns as well as the overlapping transparent patterns. The results (see Fig. 9 of Snowden et al., 1991) indicate that the suppression under the less balanced condition is indeed weaker than under the overlapping condition (there are more dots below the diagonal lines in that figure).

We discussed above that directional suppression helps to reduce noise in the visual system, and that at the same time, it makes it impossible to represent more than one motion vector in a small area. There is thus a conflict between noise reduction and multiple motion representation. The only way to represent multiple motion vectors in the absence of any other cues is to 
have spatially unbalanced motion signals in a display, such as the unpaired dot patterns. Fortunately, there are other cues present in the real world that helps to minimize the conflict. For example, different objects in the natural environment tend to exist at different depths and therefore generate different stereo disparities. Our psychophysical experiments and computer simulations have demonstrated that disparity cue can indeed help the visual system to represent two motion vectors at one location (Qian et al., 1994a, b): the paired dot patterns with a certain amount of binocular disparity introduced between the two dots in each pair is perceptually transparent. We predict, based on this observation and the results reported in this article, that the directional suppression in MT is strongest when the two different directions of motion are at the same disparity plane, and decreases as the disparity difference between the two motions increases. We are currently testing this hypothesis through singleunit recordings from $\mathrm{MT}$.

\section{References}

Adelson EH, Bergen JR (1985) Spatiotemporal energy models for the perception of motion. J Opt Soc Am [A] 2:284-299.

Allman J, Miezin F, McGuinnes E (1985) Stimulus-specific responses from beyond the classical receptive field: neurophysiological mechanisms for local-global comparisons in visual neurons. Annu Rev Neurosci 8:407-430.

Barlow HB, Levick WR (1965) The mechanism of directionally selective units in the rabbit's retina. J Physiol (Lond) 178:477-504.

Britten KH, Newsome WT (1990) Response of MT neurons to discontinuous motion. Invest Ophthalmol Visual Sci [Suppl] 31:238.

Graziano M, Andersen RA, Snowden RJ (1993) MST responses to spiral motion. J Neurosci, in press.
Qian N, Andersen RA, Adelson EH (1991) V1 responses to twosurface transparent and non-transparent motion. Soc Neurosci Abstr 17:177.

Qian N, Andersen RA, Adelson EH (1992) MT responses to transparent and non-transparent motion. Soc Neurosci Abstr 18:1101.

Qian N, Andersen RA, Adelson EH (1994a) Transparent motion perception as detection of unbalanced motion signals. I. Psychophysics. J Neurosci 14:7357-7366.

Qian N, Andersen RA, Adelson EH (1994b) Transparent motion perception as detection of unbalanced motion signals. III. Modeling. $\mathrm{J}$ Neurosci 14:7381-7392.

Reichardt W (1961) Autocorrelation, a principle for the evaluation of sensory information by the central nervous system. In: Sensory communication (Rosenblith WA, ed). New York: Wiley.

Robinson DA (1963) A method of measuring eye movement using a scleral search coil in a magnetic field. IEEE Trans Biomed Eng 10: 137-145.

Snowden RJ (1989) Motions in orthogonal directions are mutually suppressive. J Opt Soc Am [A] 7:1096-1101.

Snowden RJ, Treue S, Erickson RE, Andersen RA (1991) The response of area MT and V1 neurons to transparent motion. J Neurosci 11: $2768-2785$.

Snowden RJ, Treue S, Andersen RA (1992) The response of neurons in areas V1 and MT of the alert rhesus monkey to moving random dot patterns. Exp Brain Res 88:389-400.

Softky WR, Koch C (1993) The highly irregular firing of cortical cells is inconsistent with temporal integration of random EPSPs. J Neurosci 13:334-350.

Stoner GR, Albright TD (1991) Neural correlates of perceptual motion coherence. Nature 358:412-414.

Watson AB, Ahumada AJ (1985) Model of human visual-motion sensing. J Opt Soc Am [A] 2:322-342. 